\title{
La importancia de la evaluación de la congestión venosa por ultrasonido
}

\author{
Manuel Alberto Guerrero-Gutiérrez MD. ${ }^{1}$, \\ 1 Unidad de Cuidados Intensivos Respiratorios. Instituto Nacional de Cancerología. Ciudad de México, México.
}

Fecha de recepción: 16 de noviembre de 2021 / Fecha de aceptación: 19 de noviembre de 2021

Estimada editora,

as técnicas de imagen basadas en el ultrasonido tienen aplicaciones clínicas importantes para los pacientes críticamente enfermos que van desde pacientes ingresados en la terapia de cuidados intensivos neonatales[1], hasta el más reciente caso de pacientes con COVID-19 crítico[2]. Mención especial requiere la evaluación de la congestión venosa por ultrasonido, sobre lo cual recientemente escribimos y publicamos una revisión en la Revista Chilena de Anestesia[3]. Pese a que los trabajos originales y de revisión escritos en inglés sobre este tema son numerosos, nuestro trabajo de revisión es uno de los primeros sobre el tema que se ha publicado en español. Como ha sido resaltado por Amano y colaboradores, uno de los principales retos de la ciencia actual es la barrera del lenguaje[4], por lo cual considero de gran importancia que también difundamos el conocimiento en otros idiomas distintos al inglés.

De manera simultánea a la redacción de este artículo en español, los autores nos encontrábamos en un proyecto de redacción de un artículo en inglés sobre un tema con algunas coincidencias, pero de enfoque mucho más amplio ya que este último artículo trata sobre la importancia de tener como objetivo un balance de líquidos neutro en el paciente hospitalizado, así como de las estrategias que se pueden utilizar para ello, incluyendo una breve sección sobre la evaluación de la congestión venosa por ultrasonido, también conocido como VExUS[5]. Quiero aprovechar la oportunidad para aclarar que, aunque existe un fragmento de ambos textos que es similar en inglés y español, específicamente el que se encuentra bajo el subtítulo "¿Por qué realizar VExUS?"[3] con la sección de "Ultrasonographic evaluation of systemic venous congestión"[5], no nos fue posible citar la referencia de un artículo al otro por estar en proceso de publicación de manera simultánea. Pese a ello, ambos trabajos presentan perspectivas distintas, con distinto grado de profundización sobre los temas, por lo cual invitamos a los lectores de la Revista Chilena de Anestesia a revisar dichos artículos, que proponen una visión complementaria sobre cómo hacer mejores y más precisas evaluaciones del estado hídrico del paciente crítico.

Quiero finalmente ser enfático que en este caso no hay situación alguna de plagio, sólo contribuir con el conocimiento en castellano, como ha sido la línea editorial de su prestigiosa revista.

\section{Referencias}

1. Miller LE, Stoller JZ, Fraga MV. Point-of-care ultrasound in the neonatal ICU. Curr Opin Pediatr. 2020 Apr;32(2):216-27. https:// doi.org/10.1097/MOP.0000000000000863 PMID:31851056

2. Smith MJ, Hayward SA, Innes SM, Miller AS. Point-of-care lung ultrasound in patients with COVID-19 - a narrative review. Anaesthesia. 2020 Aug;75(8):1096-104. https://doi.org/10.1111/ anae. 15082 PMID:32275766

3. Guerrero Gutiérrez MA, Gasca Aldama JC, Pérez Nieto OR, Sánchez Díaz JS, Morgado Villaseñor LA, López Pérez FJ. Evaluación de la congestión venosa por ultrasonido. Revista Chilena de Anestesia; 50(6):825-832 https://doi.org/10.25237/revchilanestv5004101050.

4. Amano T, Rios Rojas C, Boum li Y, Calvo M, Misra BB. Ten tips for overcoming language barriers in science. Nat Hum Behav. 2021 Sep;5(9):1119-22. https://doi.org/10.1038/s41562-02101137-1 PMID:34239079

5. Pérez Nieto OR, Wong A, Lopez Fermin J, Zamarron Lopez El, Meade Aguilar JA, Deloya Tomas E, et al. Aiming for zero fluid accumulation: First, do no harm. Anaesthesiol Intensive Ther. 2021;53(2):162-78. https://doi.org/10.5114/ait.2021.105252 PMID:34006046 